\title{
An interesting case of 'skyrocketing hypercalcaemia'
}

Irfan Khan 1, Jawad Bashir 2, Shaza Ahmed 1, Fong Chau1

1 Department of Diabetes \& Endocrinology, Southmead Hospital, Bristol, UK

2 Department of Diabetes \& Endocrinology, Morriston Hospital, Swansea, UK

\section{Introduction}

Disorders of calcium metabolism are common in Sarcoidosis. The frequency of hypercalciuria and hypercalcaemia in sarcoidosis have been reported as $30-50 \%$ and $10-20 \%$ respectively. The underlying mechanism is enhanced PTH-independent extra renal production of 1,25- Dihydroxy Vitamin D (Calcitriol) which increase the absorption of calcium from the gut causing hypercalciuria and hypercalcaemia.

\section{Case report}

A 79 year old lady was admitted with fever, cough and night sweats and was treated for pneumonia. Initial investigations showed raised WCC, acute kidney injury (on the background of CKD) and normocytic anaemia. As a part of 'comprehensive geriatric assessment' she had investigations with the following results:

Corrected Calcium $2.42(2.2-2.6)$

25 (OH) Vitamin D - 14 ( $<25$ is severe deficiency)

PTH $-2.7(1.8-6.5)$

She received only one dose of $25,0001 \mathrm{U}$ Vitamin D as part of weekly replacement regime

2 days later, Calcium was 2.69 and AKI was slightly worsened

Combination of AKI, hypercalcaemia and anaemia triggered investigation for multiple myeloma which came back as normal

Over the next 3-4 days, there was further deterioration in AKI and the corrected Calcium shot up to $4.0 \mathrm{mmol} / \mathrm{L}$

Digging deep into the past medical history, the lady had a PMH of active Sarcoidosis in 1973 requiring high dose for a few years

Further investigations:

Serum ACE 88 (0-50)

HRC - No hilar lymphadenopathy or evidence of active pulmonary disease 25 (OH) Vitamin D - 16 (<25 is severe deficiency)

PTH 1.3 (1.8 - 6.5)

1,25 dihydroxy Vitamin D - $197(<143)$

\section{Discussion}

She was empirically treated with high dose Prednisolone and the Calcium level came down to normal over the next 2 weeks

This is an interesting case of acute severe hypercalcaemia

The likely underlying mechanism is extra-renal 1-alpha hydroxylation of the exogenous 25-OH Vitamin D secondary to sarcoidosis 